\title{
Performance of channels coding and space-time trellis codes on fading channel for multiple-input multiple-output free-space optical communication systems
}

\section{Ebrahim E. Elsayed ( $\nabla$ engebrahem16@gmail.com )}

Electronics and Communications Engineering Department, Faculty of Engineering, Mansoura University, Mansoura 35516, El-Dakahilia Governorate, Egypt. https://orcid.org/0000-0002-7208-2194

\section{Research Article}

Keywords: MIMO-FSO systems, space-time trellis code (STTC), space-time block codes (STBC), signal to noise ratio, atmospheric turbulence, free-space optical communications, frame error rate (FER)

Posted Date: April 26th, 2021

DOl: https://doi.org/10.21203/rs.3.rs-460147/v1

License: (c) (1) This work is licensed under a Creative Commons Attribution 4.0 International License. Read Full License 


\title{
Performance of channels coding and space-time trellis codes on fading channel for multiple-input multiple-output free-space optical communication systems
}

\author{
Ebrahim E. Elsayed
}

Electronics and Communications Engineering Department, Faculty of Engineering, Mansoura University, Mansoura 35516, El-Dakahilia Governorate, Egypt.

Author's email address: engebrahem16@gmail.com

\begin{abstract}
In this paper, we review and analyze the space-time trellis codes with and without ideal beamforming. Space-time trellis code with ideal beamforming at transmitter termed as weighted space. Smart antenna technology provides a wide variety of options, ranging from single-input multiple-output architectures that collect more energy to improve the signal-to-noise ratio at the receiver, to multiple-input multiple-output (MIMO) architectures that open up multiple data pipes over a link. Atmospheric turbulence can cause significant performance degradation in free-space optical (FSO) communication systems. An efficient solution could be to exploit the temporal diversity to improve the performance of the FSO link.
\end{abstract}

Keywords: MIMO-FSO systems, space-time trellis code (STTC), space-time block codes (STBC), signal to noise ratio, atmospheric turbulence, free-space optical communications, frame error rate (FER).

\section{Introduction}

Free-space optical (FSO) communication is a promising solution for the need for very high data rate point-to-point communication [1]. In the FSO system, the informationbearing laser beam is projected to the optical receiver along a line-of-sight. FSO has numerous advantages over radio-frequency (RF) technology [2, 3, and 4] such as very high optical bandwidth, high transmission security, and interference immunity. FSO systems have attracted considerable attention for a few years for a variety of applications and markets, e.g. last-mile connectivity, optical-fiber backup, enterprise connectivity, etc. [5]. Multiple-input multiple-output (MIMO) techniques with channel knowledge at receiver only are generally of two types, spatial multiplexing systems, and diversity systems. Spatial multiplexing or Bell labs layered space-time (BLAST) type systems $[2,6]$, on the other hand, transmit independent data streams from each transmitting antenna, permitting spectral efficiency to be attained at the cost of a loss in diversity advantage for a fixed number of receive antennas. Diversity modulation, or space-time coding [3-8], uses code words designed to maximize the 
diversity advantage of the transmitted information. The signaling strategy also depends on the availability of channel information at the transmitter. For example, MIMO does not require channel knowledge at the transmitter, although it enjoys improved performance if channel information is available [9-12]. The main techniques employed for MIMO channels to offer spatial diversity is space-time block codes (STBC) [6] and space-time trellis codes (STTC) [7, 12-14]. Both offer full spatial diversity. Space-time block codes have no coding gain. Therefore, they are typically concatenated with an outer channel code separated by an interleaver ensuring locally uncorrelated bits for modulation [14-16].

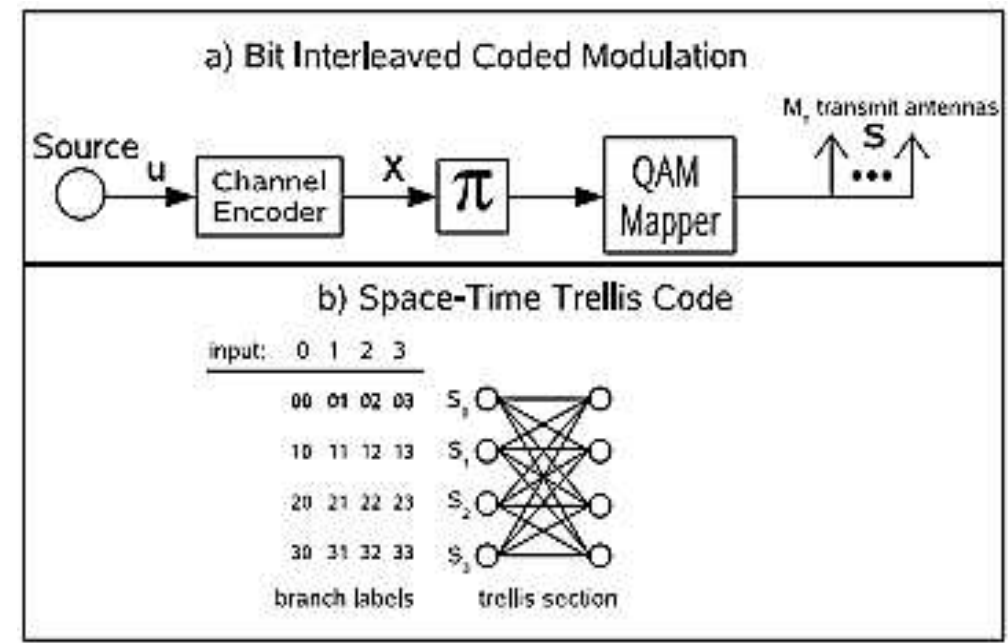

Fig. 1: State-of-the-art encoder: a) bit interleaved coded modulation b) space-time trellis code for 4-QAM, 4-states, and 2 transmit antennas.

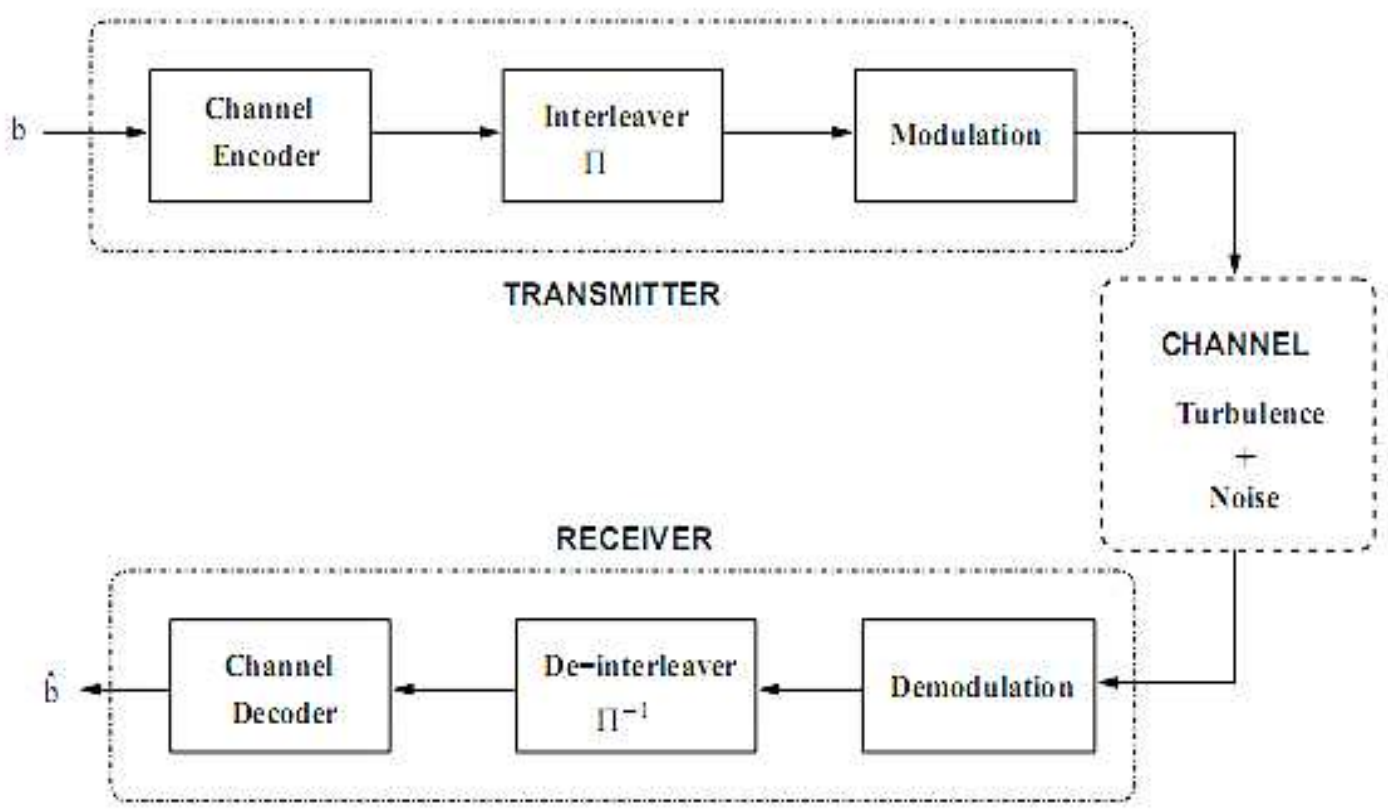

Fig. 2: System block diagram [16]. 


\section{System model}

Figure 1 shows the state-of-the-art encoder: a) bit interleaved coded modulation b) Space-time trellis code for 4-QAM, 4-states, and 2 transmit antennas. The general system block diagram is given in Fig. 2. Each of the three main blocks of transmitter channel and receiver is described in the following.

\subsection{Transmitter}

The encoded bits are then interleaved before being converted to symbols (the modulation block) and transmitted through the channel. We consider pseudorandom interleaving in this work by which the encoded bits are permuted in a random manner (but known at the receiver) [14]. Also, we consider the most commonly used intensity modulation technique, that is, the non-return-to-zero onoff keying. So, the presence of an impulse of duration $T_{S}$ represents a binary bit 1 (On state), whereas the absence of signal for the same duration represents a binary bit 0 (Off state) [12-15].

\subsection{Channel modeling}

We consider the time variations according to the theoretical block-fading model, where the channel fade remains constant during a block (corresponding to the channel coherence interval) and changes to a new independent value from one block to the next [8-10]. Let us denote by $h_{x}$ and $h_{y}$ the large-and small-scale irradiance fluctuations, respectively. $h_{x}$ and $h_{y}$ are assumed to be statistically independent and described by a Gamma distribution. Based on these assumptions, the intensity fluctuation $\mathrm{h}=h_{x} \quad h_{y}$ has Gamma-Gamma distribution with the probability density function (PDF) as follows [16-19].

$P_{G G}\left(h_{X}\right)=\frac{2(\alpha \beta)^{(\alpha+\beta)} / 2}{\Gamma(\alpha) \Gamma(\beta)} h_{X}^{(\alpha+\beta) / 2-1} K_{\alpha-\beta}\left(2 \sqrt{\alpha \beta h X)} ; h_{X}>0, \quad\right.$ Eq. 1

$\alpha=\left\{\exp \left[\frac{0.49 \sigma_{R}^{2}}{\left(1+0.65 d^{2}+1.11 \sigma_{R}^{12 / 5}\right)^{\frac{7}{6}}}\right]-1\right\}^{-1}$ 
$\beta=\left\{\exp \left[\frac{0.51 \sigma_{R}^{2}\left(1+0.69 \sigma_{R}^{12 / 5}\right)^{-5 / 6}}{1+0.9 d^{2}+0.62 d^{2} \sigma_{R}^{12 / 5}}\right]-1\right\}^{-1}$

Eq. 3

Where $\mathrm{d}=\sqrt{K D_{R X}^{2} / 4 l_{f s o}}$ is the normalized $\mathrm{RCL}$ radius, $\sigma_{R}^{2}=1.23 C_{n}^{2} K^{7 / 6} l_{f s o}^{11 / 6}$ is the Rytov variance [18], $\mathrm{C}_{\mathrm{n}}^{2}$ is the refractive index structure constant (ranging from $10^{-17} \mathrm{~m}^{-2 / 3}$ to $\left.\sim 10^{-13} \mathrm{~m}^{-2 / 3}\right), l_{\text {fso }}$ is the FSO link length, $\mathrm{K}=2 \pi / \lambda$ is the wavenumber [17].

\subsection{Receiver}

At the receiver, after signal detection (the demodulator block) and de-interleaving, channel decoding is performed (see Fig. 2). The demodulation is performed based on the received signal light intensity. The electrical signal after the optical/ electrical conversion is [16]:

$r_{e}=\eta\left(I+I_{a}\right) n$

Eq. 4

where $\mathrm{I}$ is the received signal light intensity, $I_{a}$ is the remaining ambient light intensity after frequency and spatial filtering. So, after the cancellation of the ambient light, the received signal before demodulation is [16-18]:

$r=\eta I+n$

Eq. 5

And the noise variance equals

$\sigma_{n}^{2}=\mathrm{N}_{0} / 2 \mathrm{~T}_{\mathrm{S}}$

Eq. 6 
The received signal intensity I as the product of $I_{0}$, the emitted light intensity, and $h^{\prime \prime}$, the channel atmospheric turbulence with the PDF given in (18) :

$\mathrm{I}=h I_{0}$

The demodulator provides at its output logarithmic likelihood ratios on the transmitted bits instead of hard values 0 or 1 .

\section{Space-time coding}

Space-time coding (STC) [3] exploits the diversity provided by the MIMO channel in both spaces (antenna) and time domains, thus significantly increasing the system capacity as well as improving the reliability of the wireless link. STTC is a class of signaling techniques that conglomerate the design of the channel code with transmitting and optionally receive antenna diversity. Furthermore, to the diversity advantage, a certain amount of coding gain can be attained by a well-designed STTC. STTCs use a trellis encoder to host redundancy into the transmitted symbol stream and to accomplish coding gain $[10,16]$. The coding gain is reliant on the length of the memory in the encoder and on the construction criteria of the code. A number of several structures have been projected for STTCs [13].

\section{Space-time trellis code}

In 1998, V. Tarokh, H. Jafarkhani, and A. R. [5] proposed the model of STTCs using multiple transmit antennas. STTCs can concurrently provide substantial coding gain, spectral efficiency, and diversity improvement. But with the increase in the number of antennas and the size of the modulation, there is an exponential increase in decoding complexity set is a major drawback [5-8]. MIMO system with $n_{R}$ receive antennas and $\mathrm{n}_{\mathrm{T}}$ transmits antennas as shown in Fig. 3. 


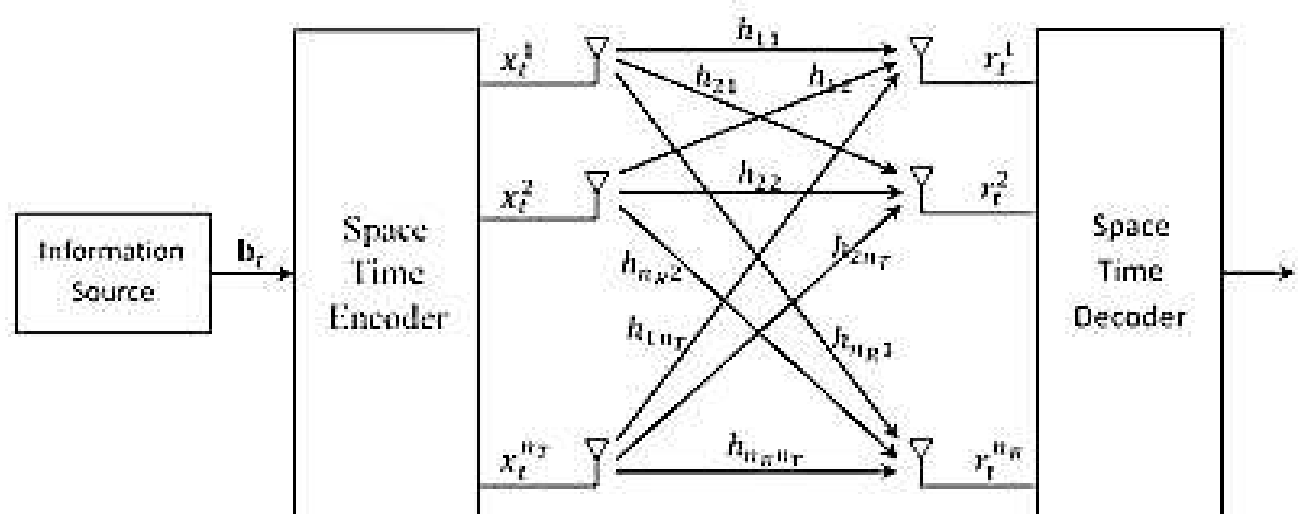

Fig. 3: Block diagram of a MIMO system.

The signal at each $n_{R}$ receive antennas is a noisy superposition of the $\mathrm{n}_{\mathrm{T}}$ transmitted signals infected by channel fading. The received signal at antenna $j$ where $j=1,2, n_{R}$ at the time represented by $r_{t}^{j}$ is given by $[10,14]$,

$\mathrm{r}_{\mathrm{t}}^{\mathrm{j}}=\sum_{\mathrm{i}=1}^{\mathrm{n}_{\mathrm{T}}} \mathrm{h}_{\mathrm{j}, \mathrm{i}}^{\mathrm{t}} \mathrm{x}_{\mathrm{t}}^{\mathrm{i}}+\mathrm{n}_{\mathrm{t}}^{\mathrm{j}}$

Thus, the received signal vector can be represented as [10],

$\mathrm{r}_{\mathrm{t}}=\mathrm{H}_{\mathrm{t}} \mathrm{x}_{\mathrm{x}}+\mathrm{n}_{\mathrm{t}}$

We suppose that the decoder at the receiver uses the Viterbi algorithm to achieve maximum likelihood (ML) decoding and that the receiver has ideal channel state information on the MIMO channel [10-15]. The transmitter has no information about the channel. At the receiver, the decision metric is calculated based on squared Euclidean distance between the theorized received sequence and the received sequence as [16-19],

$\mathrm{x}_{\mathrm{t}}^{\mathrm{i}}=\sum_{\mathrm{k}=1}^{\mathrm{m}} \sum_{\mathrm{j}=0}^{\mathrm{U}_{\mathrm{K}}} \mathrm{g}_{\mathrm{j}, \mathrm{i}}^{\mathrm{k}} \mathrm{b}_{\mathrm{t}-\mathrm{j}}^{\mathrm{k}} \quad \bmod \mathrm{M}$

Eq. 10

$\mathrm{U}=\sum_{\mathrm{k}=1}^{\mathrm{m}} \mathrm{U}_{\mathrm{k}}$

$\bmod \mathrm{M}$

Eq. 11

5. Viterbi algorithm for STTC.

The Viterbi algorithm chooses the path with the minimum path metric as the decoded sequence [10]. The spectral efficiency of the system is [14-16],

$\mathrm{H}=\frac{\mathrm{r}_{\mathrm{b}}}{\mathrm{B}}=\mathrm{m} \frac{\mathrm{bits}}{\mathrm{sec}} / \mathrm{Hz}$ 
6. Near-maximum-likelihood Viterbi decoding of the space-time bit trellis codes (STbitTC).

Due to their binary trellis structure, STbitTCs offer a high flexibility regarding modulation and antenna constellations. In the following section, we focus on the decoding for the encoder in Fig. 2. This new symbol trellis has the same number of states and a length of $N=N C / Q$ steps (see Fig. 3). As the code structure has not been broken by an interleaver only $2^{R Q}$ transitions have to be considered for each state instead of $2^{Q}$.

\section{Simulation results}

We evaluate the effect of receive diversity on the error performance of the code. Performance is calculated by using 2 transmit antennas and a different number of receive antennas for the Rayleigh fading channel [19-24]. The performance of a weighted space-time trellis code (WSTTC) system for the Rayleigh fading channel is given by plotting frame-error-rate (FER) against the signal-to-noise ratio [23-26]. The FER performances for the 4-QPSK constellation, STTC system for the Rayleigh fading channel are shown in Fig. 4 . The Performance is calculated with 2 transmits antennas i.e., and a different number of receive antennas for $n_{r}=1,2,4$. Fig. 5 shows the average received irradiance against bit-error-rate (BER) for all cases: (a) quantum limit case (b) thermal noise case (c) background radiation case (d) background and thermal noise case. The BER of the binary pulse position modulation for FSO under the weak turbulence is shown in Fig. 6 . Fig. 7 shows the BER for the binary phaseshift keying modulation in a Rayleigh fading channel with selection diversity. Fig. 8 shows the $E b / N_{0}(d B)$ versus the optical STTC coding FSO communication space-time trellis code, introducing inverse fast Fourier transforms, joined the DC offset, used the Viterbi decoding algorithm. The Eb/ $\mathrm{N}_{0}(\mathrm{~dB})$ vs. BER by optical STTC coding FSO link space-time trellis code is shown in Fig. 9. Also, the $E b / N_{0}(d B)$ vs. the BER by optical WSTTC coding FSO communication weighted space-time trellis code is shown in Fig. 10. Finally, Fig. 11 shows the Eb/N $\mathrm{N}_{0}(\mathrm{~dB})$ vs. BER for near-ML Viterbi decoding of STbitTC. The simulation result illustrates that the STC, STTC, STBC, WSTTC, and near-ML Viterbi decoding of STbitTC are worth to be implemented on the wireless communication systems 


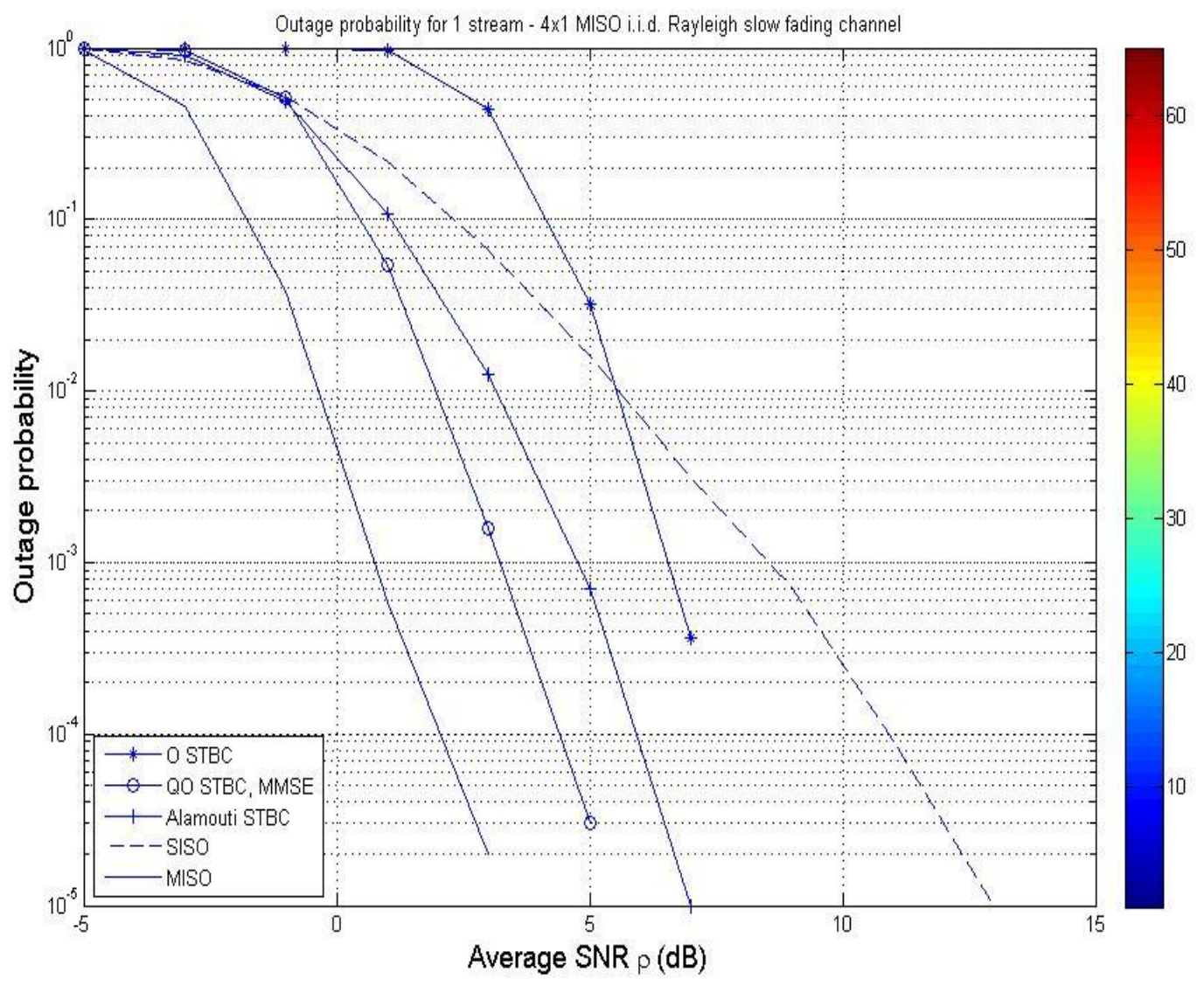

Fig. 4: Communications performance for $4 \times 4$ antenna system with $16-\mathrm{QAM}$ and $R=1 / 2$ STbitTC decoding $(\eta)=8$ bit per channel use). 

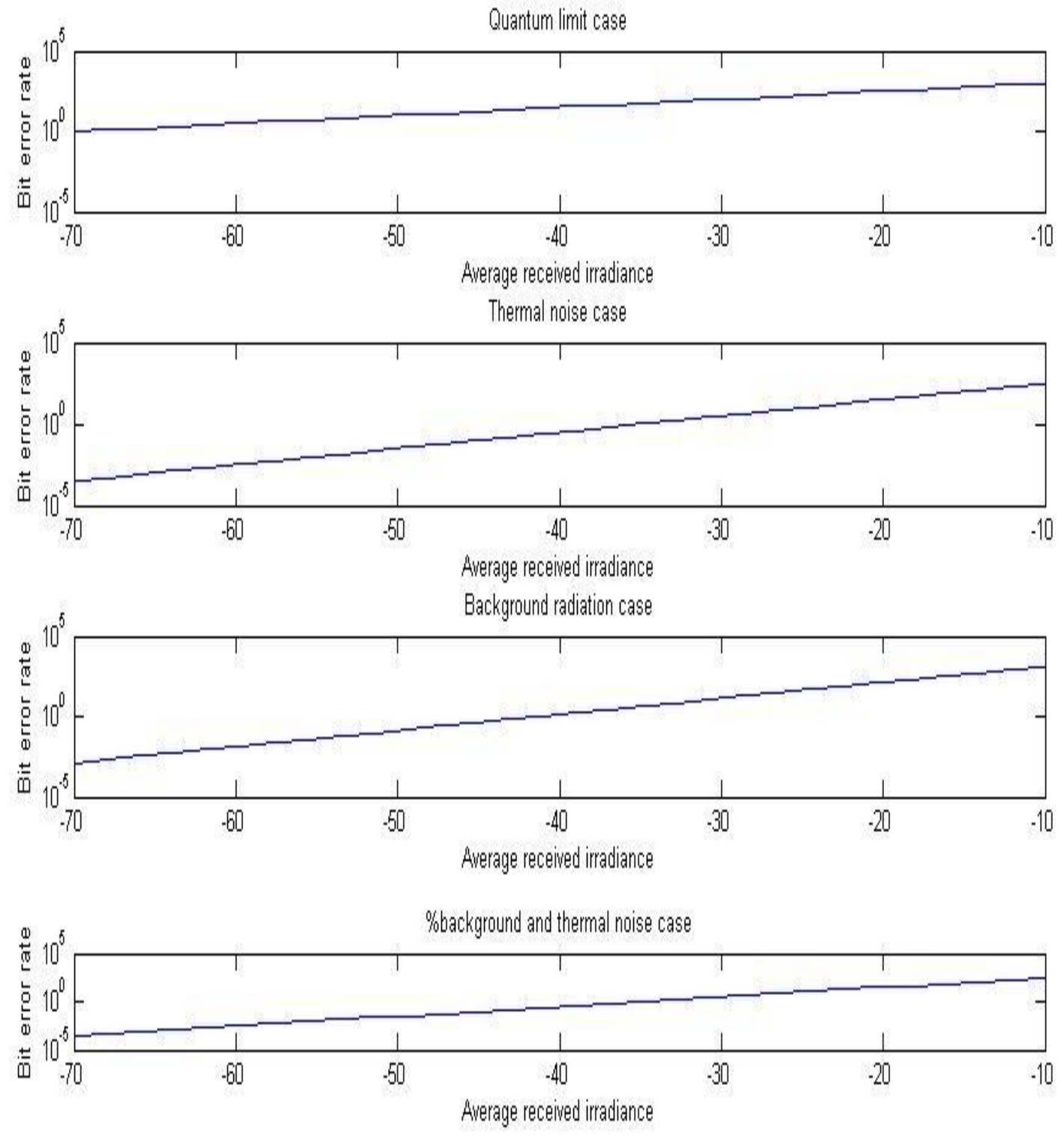

Fig. 5: Average received irradiance against the BER for all cases: (a) quantum limit case (b) thermal noise case (c) background radiation case (d) background and thermal noise case. 


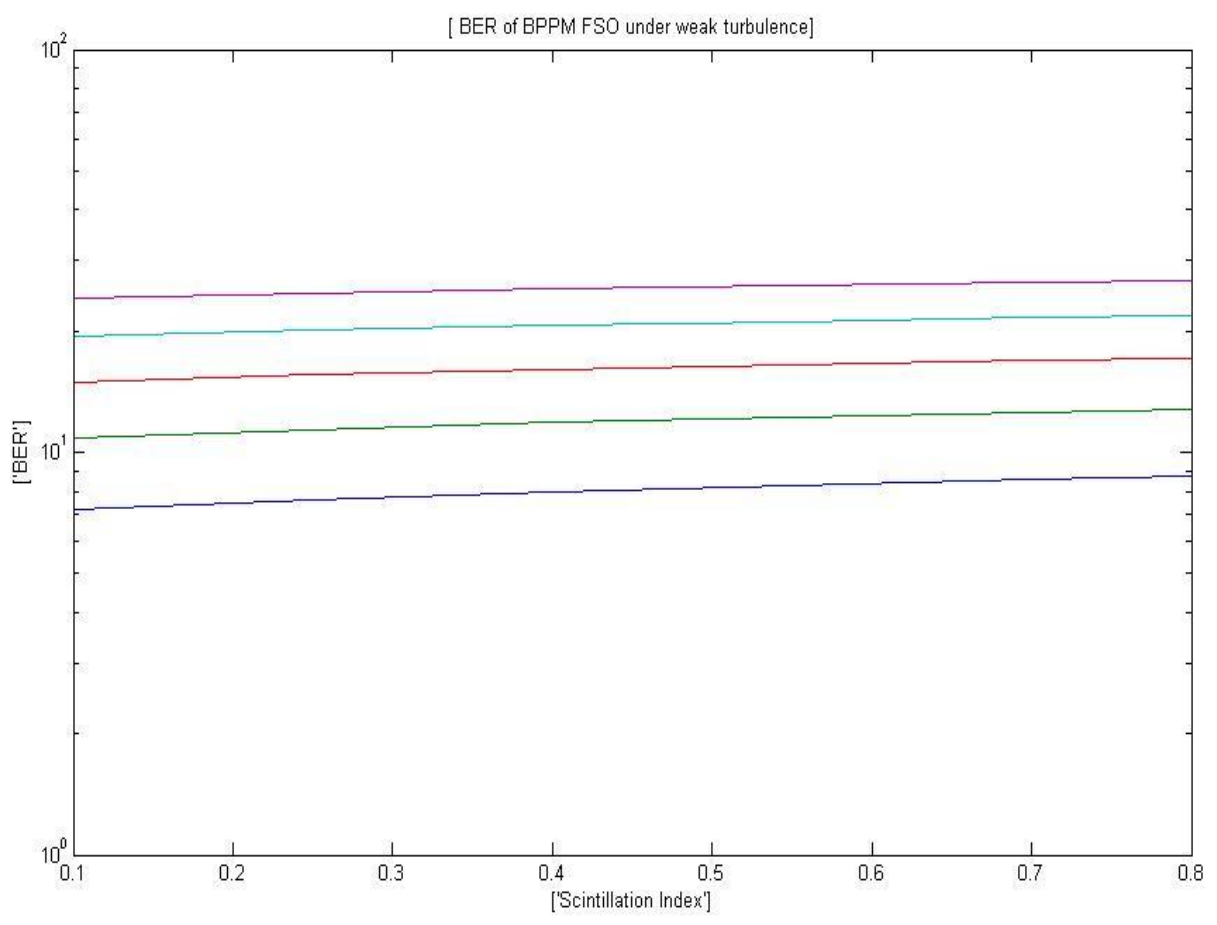

Fig. 6: BER of the binary pulse position modulation for FSO under the weak turbulence.

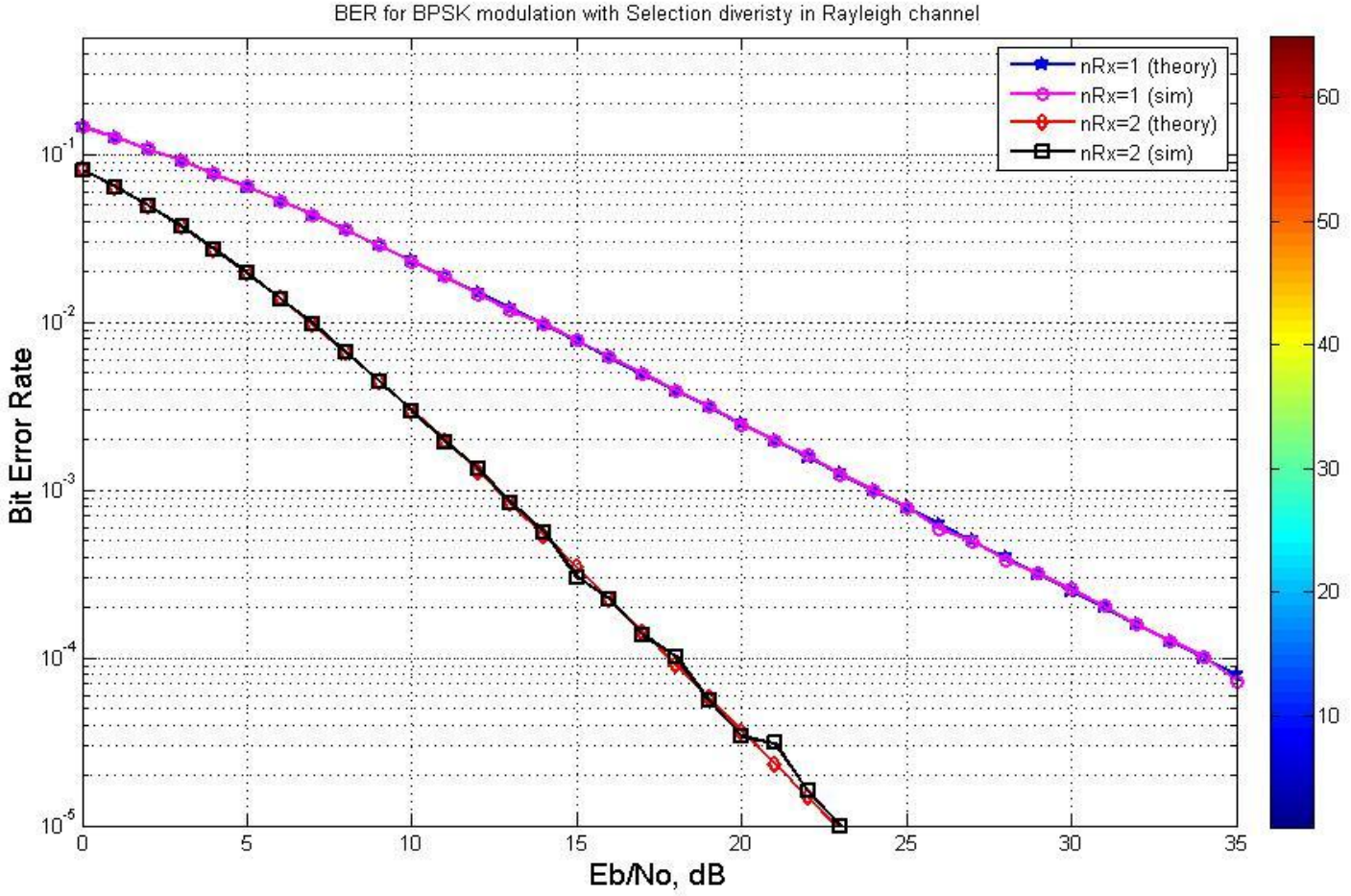

Fig. 7: BER for the binary phase-shift keying modulation in a Rayleigh fading channel with selection diversity. 


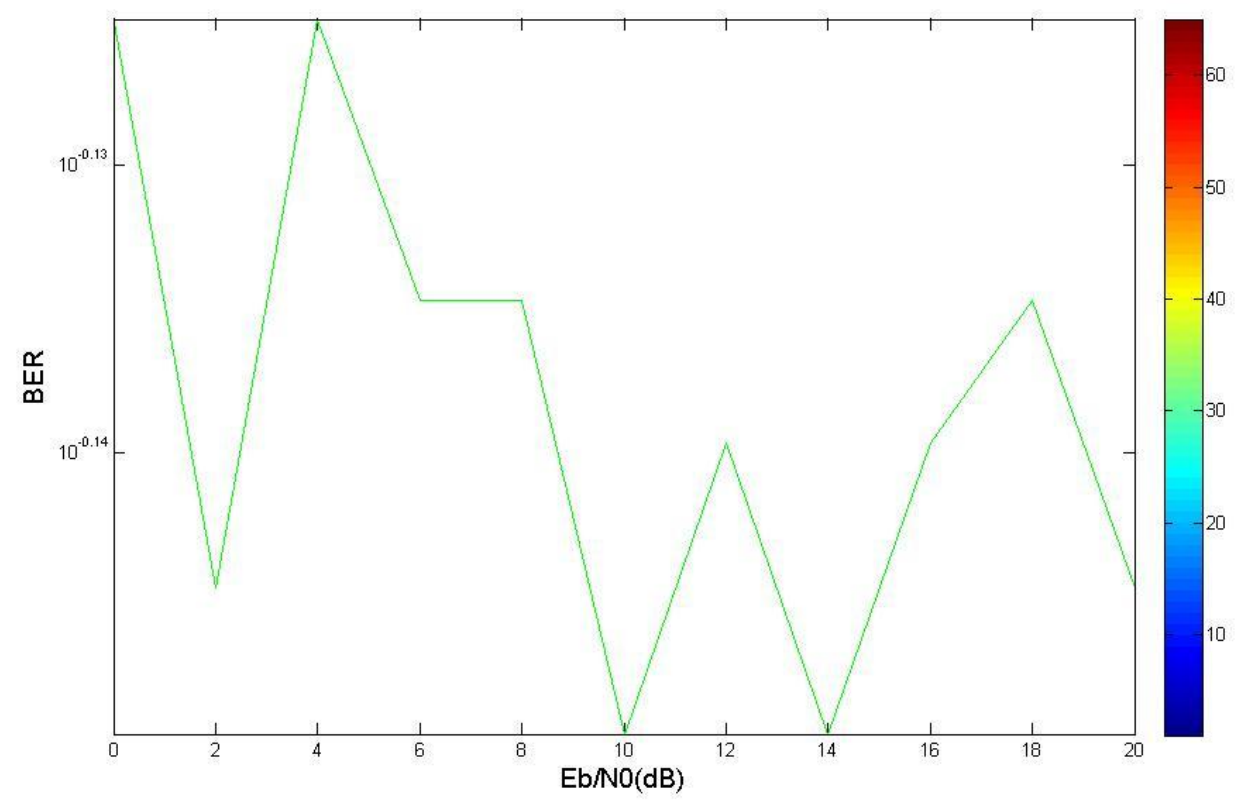

Fig. 8: $\mathrm{Eb} / \mathrm{N}_{0}(\mathrm{~dB})$ vs. the BER optical STTC coding Description FSO communication space-time trellis code, introducing inverse fast Fourier transform, joined the DC offset, used Viterbi decoding algorithm.

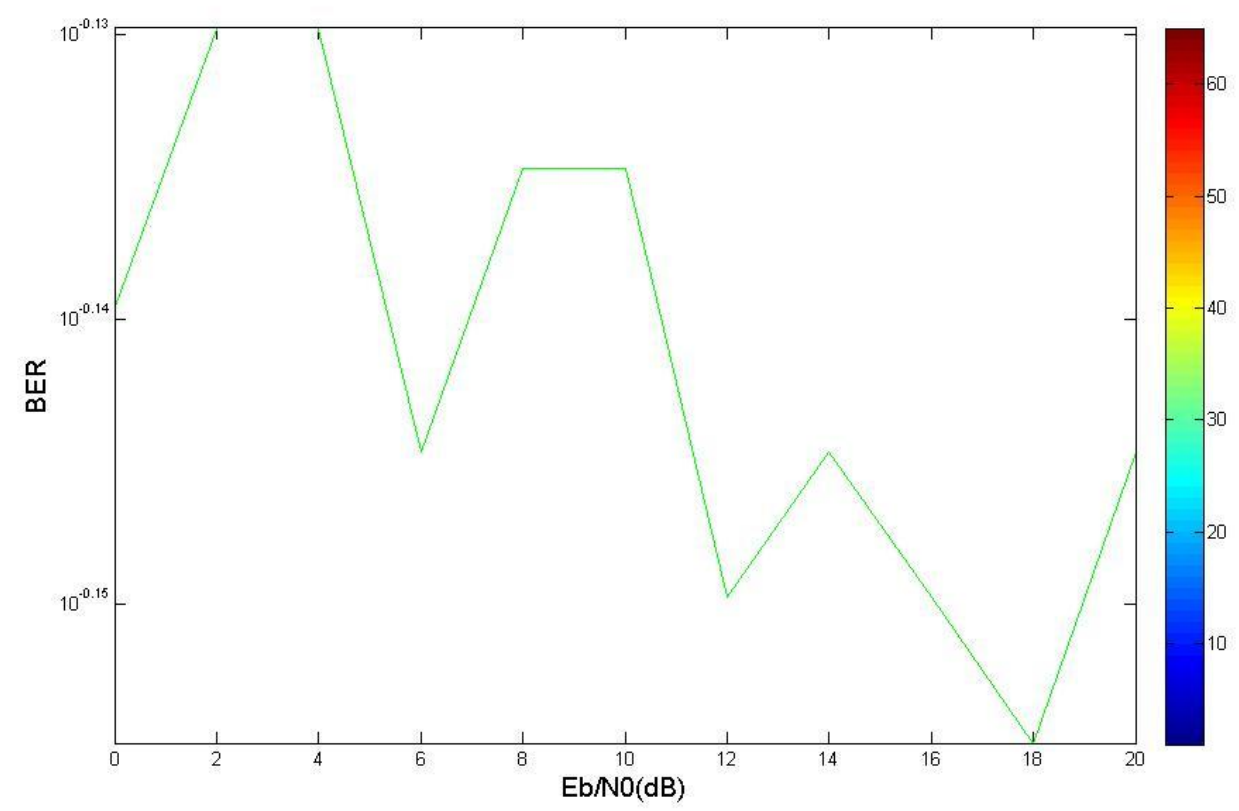

Fig. 9: $E b / N_{0}(d B)$ vs. the BER by optical STTC coding free-space optical link spacetime trellis code. 


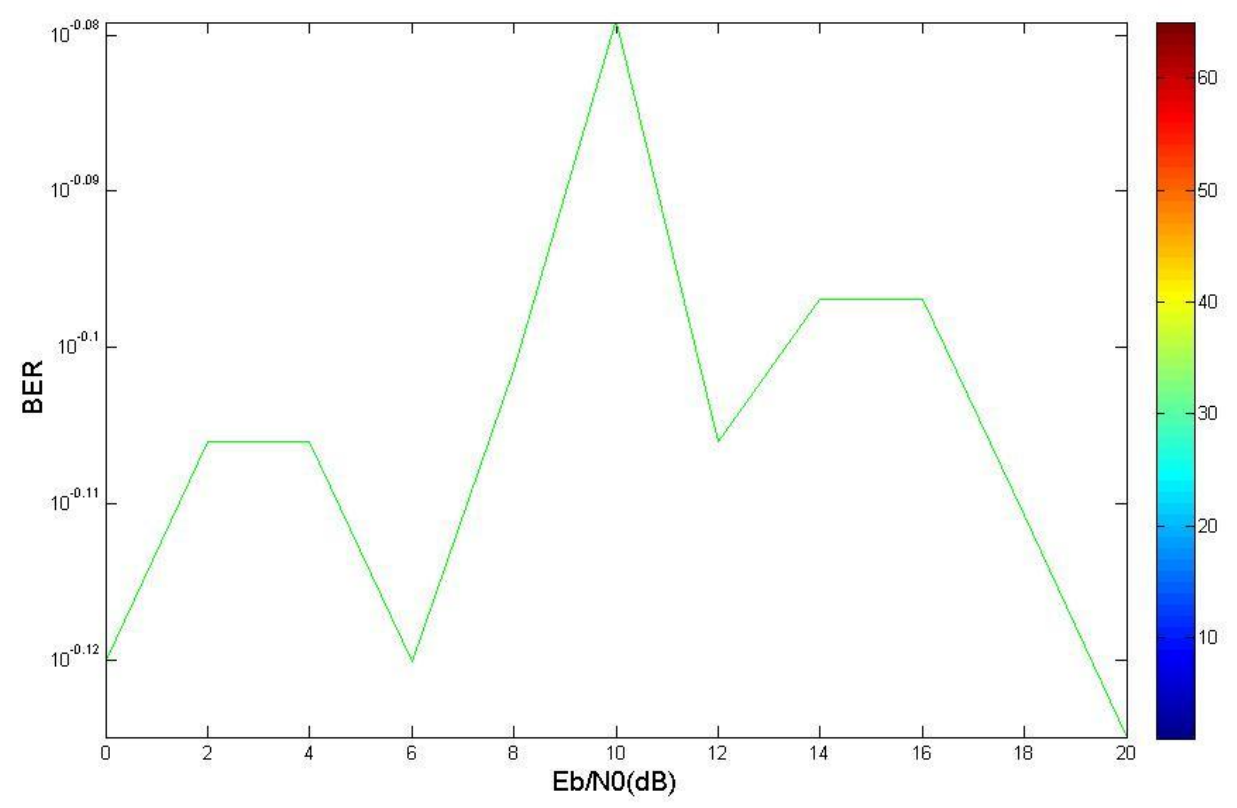

Fig. 10: $\mathrm{Eb} / \mathrm{N}_{0}(\mathrm{~dB})$ vs. the BER by Optical WSTTC coding FSO communication weighted space-time trellis code.

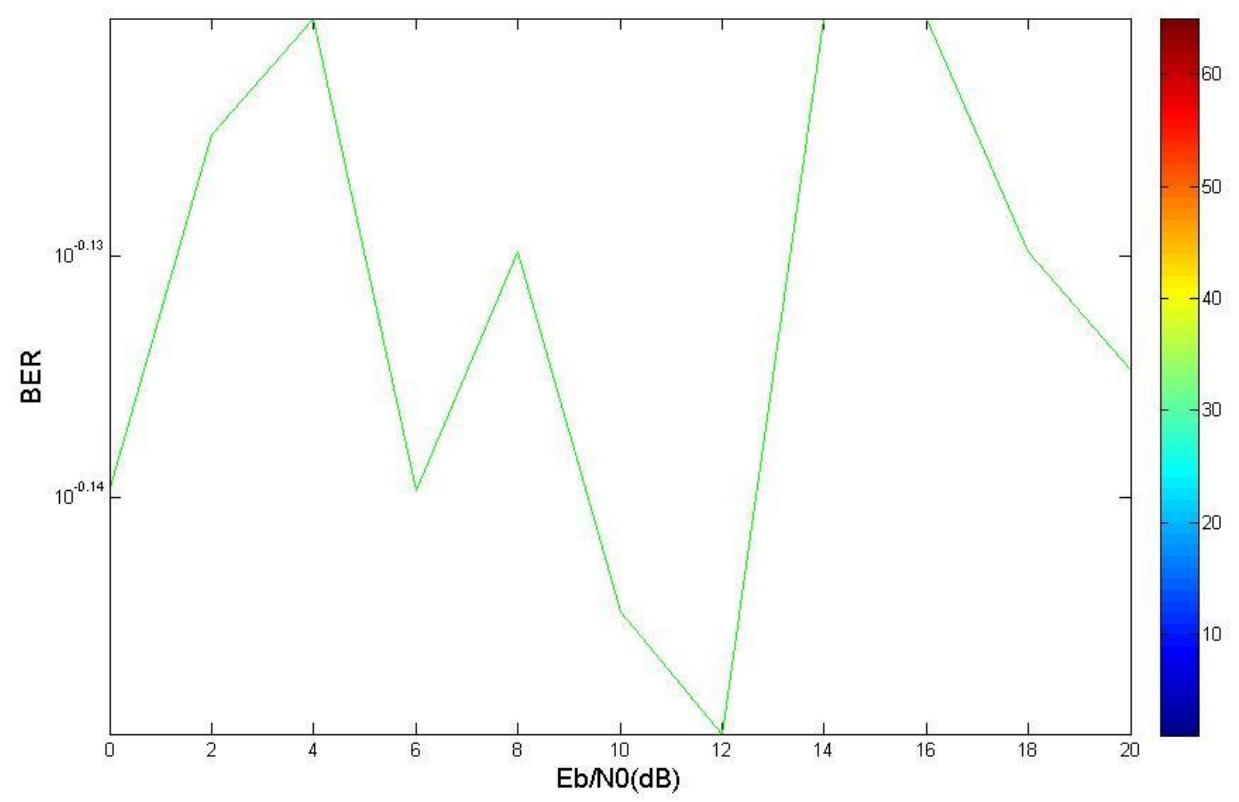

Fig. 11: $E b / N_{0}(d B)$ vs. the BER for near-ML Viterbi decoding of STbitTC. 


\section{Conclusions and discussion}

For FSO transmission link submitted to different turbulence conditions, we performed a comparative study of the performances of different channel coding techniques, employed under the presence or not of time diversity. The simulation result illustrates that the STC, STTC, STBC, WSTTC, and near-ML Viterbi decoding of STbitTC are worth to be implemented on the wireless communication systems. As they are providing far less bit-error-rate as compare to no coding communication. An increase in the number of transmitters and receivers antennas enhances the performance of STTC and STBC.

\section{References}

1. Goldsmith, Wireless Communications, Cambridge University Press, 2005.

2. G. J. Foschini, "Layered space-time architecture for wireless communication in a fading environment when using multi-element antennas," Bell Labs Technical Journal, vol. 1, no. 2, pp. 41-59, Jun 1996.

3. H. Jafarkhani, Space-time coding: theory and practice, Cambridge university press, 2005.

4. J. H. Winters, "The diversity gain of transmit diversity in wireless systems with Rayleigh fading," IEEE Transactions on Vehicular Technology, vol. 47, no. 1, pp. 119-123, 1998.

5. V. Tarokh, N. Seshadri and A. Calderbank, "Space-time codes for high data rate wireless communication: performance criterion and code construction," IEEE Transactions on Information Theory, vol. 44, no. 2, pp. 744-765, Mar 1998.

6. S. M. Alamouti, "A simple transmit diversity technique for wireless communications," IEEE Journal on Selected Areas in Communications, vol. 16, no. 8, pp. 1451-1458, 1998.

7. IEEE802.11n, "Wireless LAN Medium Access Control and Physical Layer specifications: Enhancements for Higher Throughput,"IEEEP802.11n/D3.0, Sept. 2007.

8. S. M. Alamouti, "A simple transmit diversity technique for wireless communications," Selected Areas in Communications, IEEE Journal on, vol. 16 , no. 8, pp. 1451-1458,1998.

9. J. Hagenauer and C. Kuhn, "The List- Sequential (LISS) Algorithm and Its Application," IEEE Transactions on Communications, vol. 55, no. 5, pp. 918928, May 2007.

10. G. Fettweis and H. Meyr, "High-Speed Parallel Viterbi Decoding: Algorithm and VLSI-Architecture," IEEE Communications Magazine, vol. 29, pp. 46-55, May 1991. 
11. S. Lin and D. J. Costello Jr, Error Control Coding 2nd., Prentice Hall PTR, Upper Saddle River, New Jersey, USA, 2004.

12. M. O. Damen, H. El Gamal, and G. Caire, "On maximum-likelihood detection and the search for the closest lattice point," IEEE Transactions on Information Theory, vol. 49, no. 10, pp. 2389-2402, Oct. 2003.

13. A. Burg, M. Borgmann, M. Wenk, M. Z. ellweger, W. Fichtner, and H. Bolcskei," VLSI implementation of MIMO detection using the sphere decoding algorithm," IEEE Journal of Solid-State Circuits, vol. 40, no. 7, pp. 1566-1577, 2005.

14. V. Tarokh, N. Seshadri, and A. R. Calder bank, "Space-time codes for high data rate wireless communication: performance criterion and code construction," IEEE Transactions on Information Theory, vol. 44, pp. 744-765, 1998.

15. S. Baro, G. Bauch, and A. Hansmann, "Improved codes for space-time trelliscoded modulation," IEEE Communications Letters, vol. 4, no. 1, pp. 20-22, Jan. 2000.

16. Fang $\mathrm{Xu}$, Ali Khalighi, Patrice Caussee, and Salah Bourennane "Channel coding and time-diversity for optical wireless links", (C2008 Optical Society of America OCIS codes: 060.2605, Free-space optical communication, 060. 4510 Optical communications.

17. M. A. Al- Habash, L. C. Andrews and R. L. Phillips, "Mathematical model for the irradiance probability density function of a laser beam propagating through turbulent media, "Opt. Eng. 40, 1554-1562 (2001).

18. V. W. S. Chan, "Free-space optical communications," J. Lightwave Technol. 24, 4750-4762 (2006).

19. L. C. Andrews and R. L. Phillips, Laser Beam Propagation through Random Media (SPIE Press, Bellingham, Washington, 2005), 2nded.

20. E. E. Elsayed and B. B. Yousif, "Performance evaluation and enhancement of the modified OOK based IM/DD techniques for hybrid fiber/FSO communication over WDM-PON systems," Opt. Quantum Electron., vol. 52, no. 9, 2020, doi: 10.1007/s11082-020-02497-0.

21. B. B. Yousif, E. E. Elsayed, and M. M. Alzalabani, "Atmospheric turbulence mitigation using spatial mode multiplexing and modified pulse position modulation in hybrid RF/FSO orbital-angular-momentum multiplexed based on MIMO wireless communications system," Opt. Commun., vol. 436, pp. 197-208, 2019, doi: 10.1016/j.optcom.2018.12.034.

22. B. B. Yousif and E. E. Elsayed, "Performance Enhancement of an OrbitalAngular-Momentum-Multiplexed Free-Space Optical Link under Atmospheric Turbulence Effects Using Spatial-Mode Multiplexing and Hybrid Diversity Based on Adaptive MIMO Equalization," IEEE Access, vol. 7, pp. 84401-84412, 2019, doi: 10.1109/ACCESS.2019.2924531. 
23. E. E. Elsayed and B. B. Yousif, "Performance enhancement of hybrid diversity for M-ary modified pulse-position modulation and spatial modulation of MIMO-FSO systems under the atmospheric turbulence effects with geometric spreading," Opt. Quantum Electron., vol. 52, no. 12, 2020, doi: 10.1007/s11082-020-02612-1.

24. Ebrahim E. Elsayed, Bedir B. Yousif, and Mahmoud M. Alzalabani, "Performance enhancement of the power penalty in DWDM FSO communication using DPPM and OOK modulation", Optical and Quantum Electronics, vol. 50 (7), pp. 282, (26 June 2018).

25. Ansari, N., Zhang, J.: 'Media access control and resource allocation for next generation passive optical networks' (Springer, 2013)

26. Ebrahim E. Elsayed and Bedir B. Yousif, "Performance enhancement of the average spectral efficiency using an aperture averaging and spatial-coherence diversity based on the modified-PPM modulation for MISO FSO links", Optics Communications, vol. 463, pp. 125463, (15 May 2020).

\section{COMPLIANCE WITH ETHICAL STANDARDS}

Conflict of interest: The author declares that there is no conflict of interest regarding the manuscript. The author is responsible for the content and writing of this article. The author declares that he has no known competing financial interests or personal relationships that could have appeared to influence the work reported in this paper. 
Figures

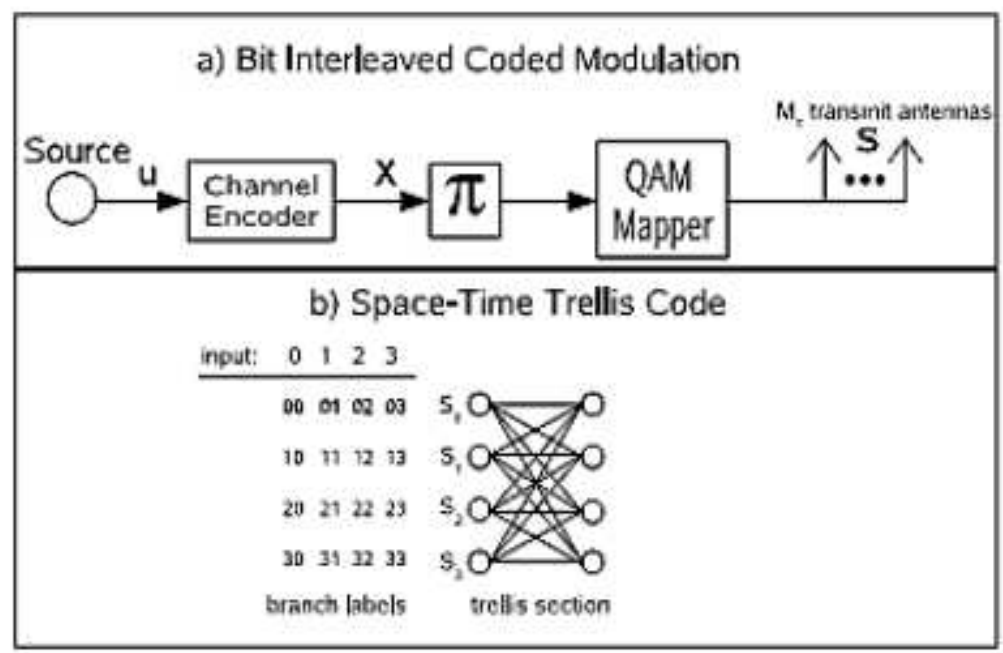

\section{Figure 1}

State-of-the-art encoder: a) bit interleaved coded modulation b) space-time trellis code for 4-QAM, 4states, and 2 transmit antennas.

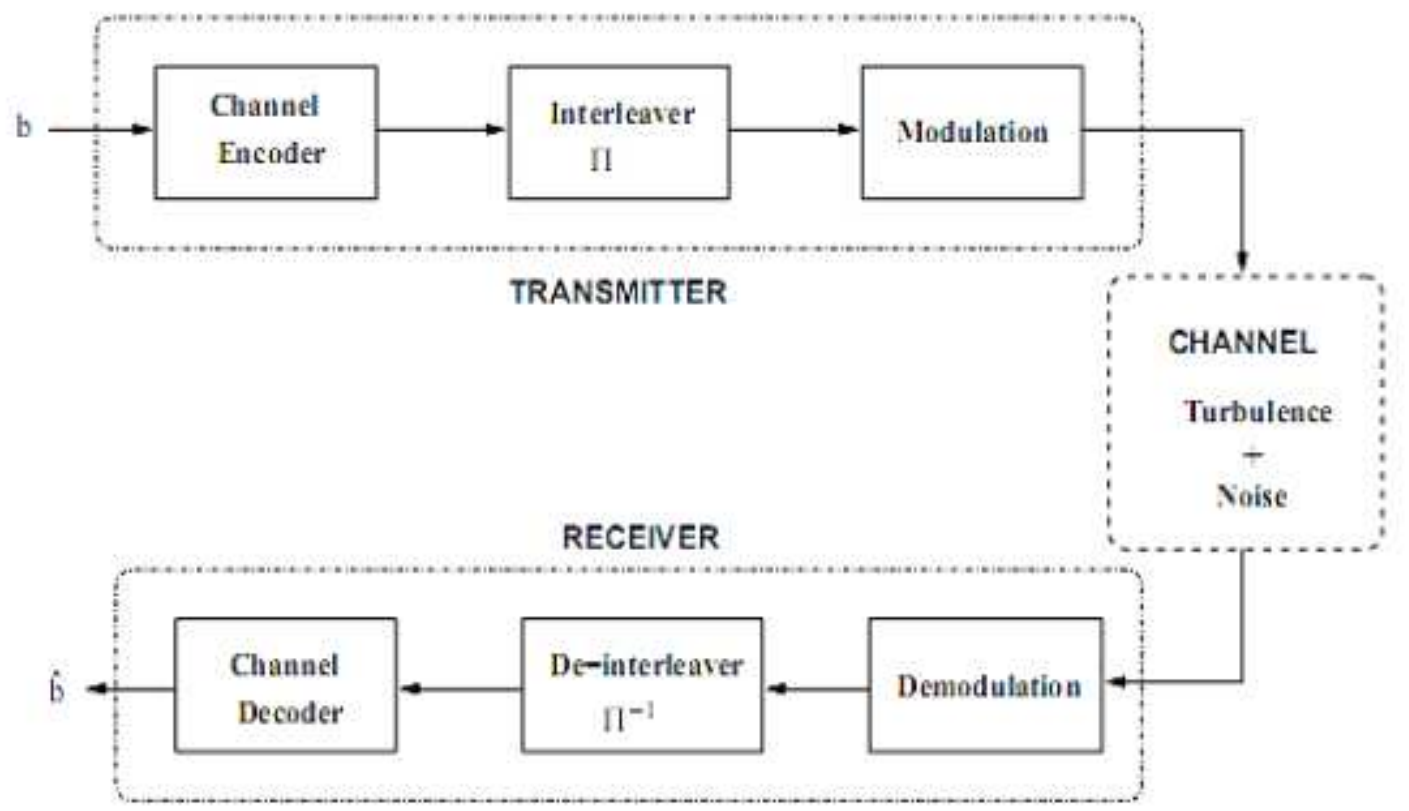

\section{Figure 2}

System block diagram [16]. 


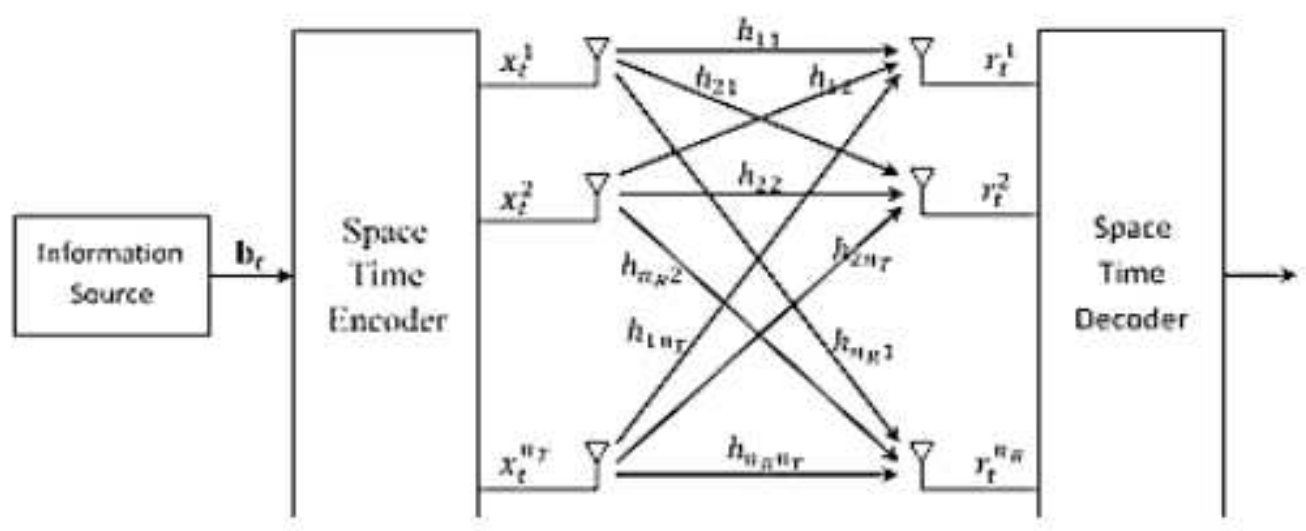

Figure 3

Block diagram of a MIMO system.

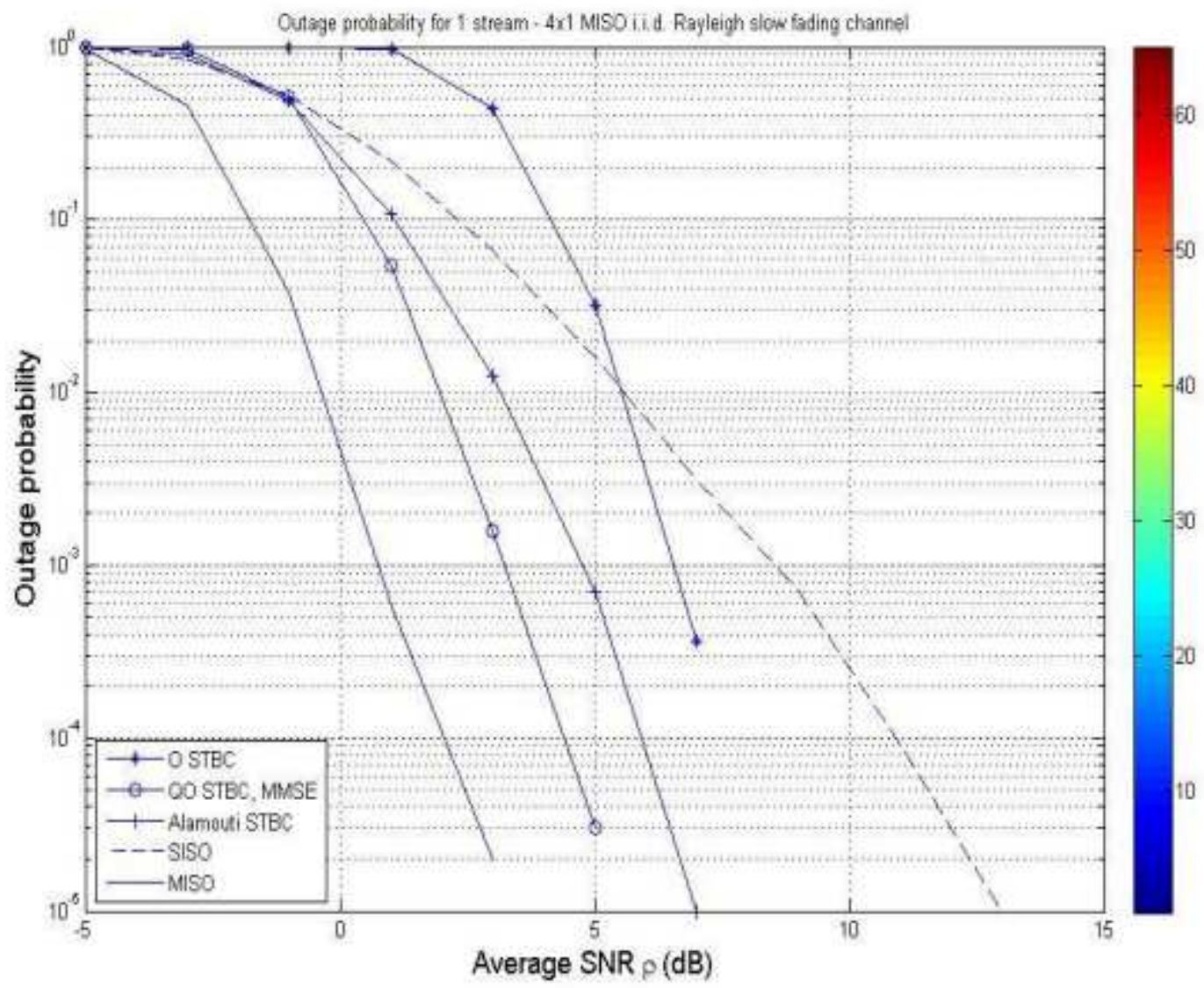

\section{Figure 4}

Communications performance for 44 antenna system with 16-QAM and R 1/2 S bit C decoding $\eta$ ) = 8 bit per channel use). 

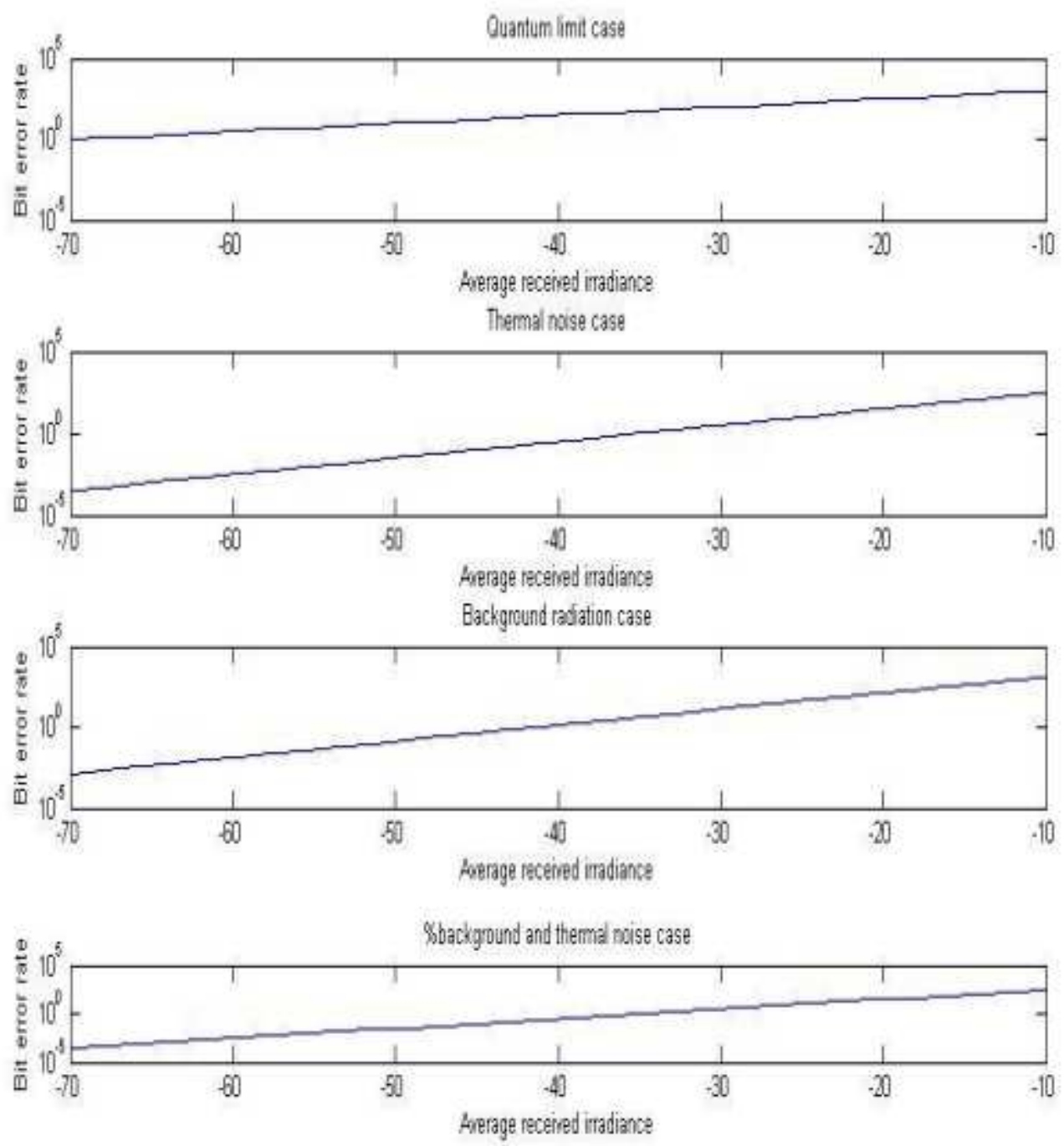

\section{Figure 5}

Average received irradiance against the BER for all cases: (a) quantum limit case (b) thermal noise case (c) background radiation case (d) background and thermal noise case. 


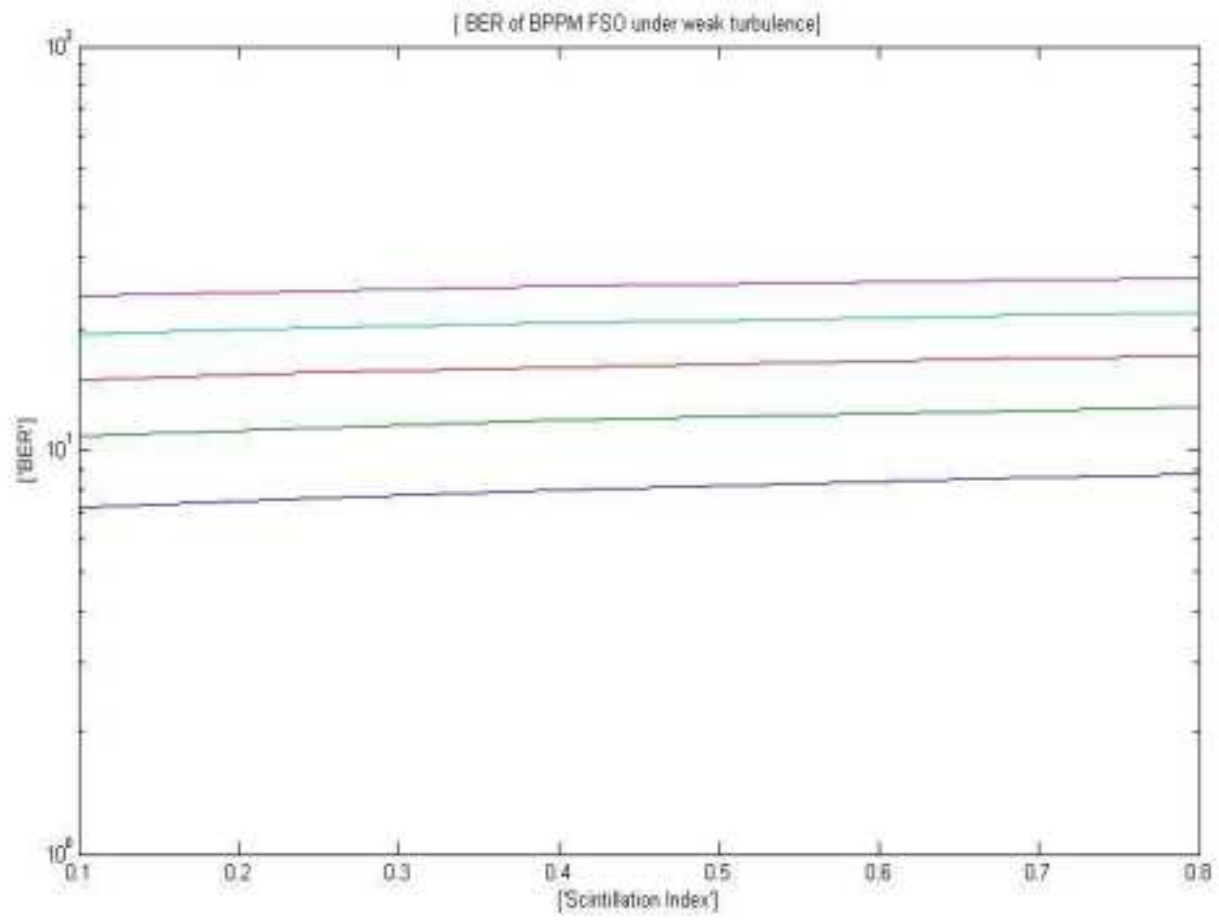

Figure 6

BER of the binary pulse position modulation for FSO under the weak turbulence.

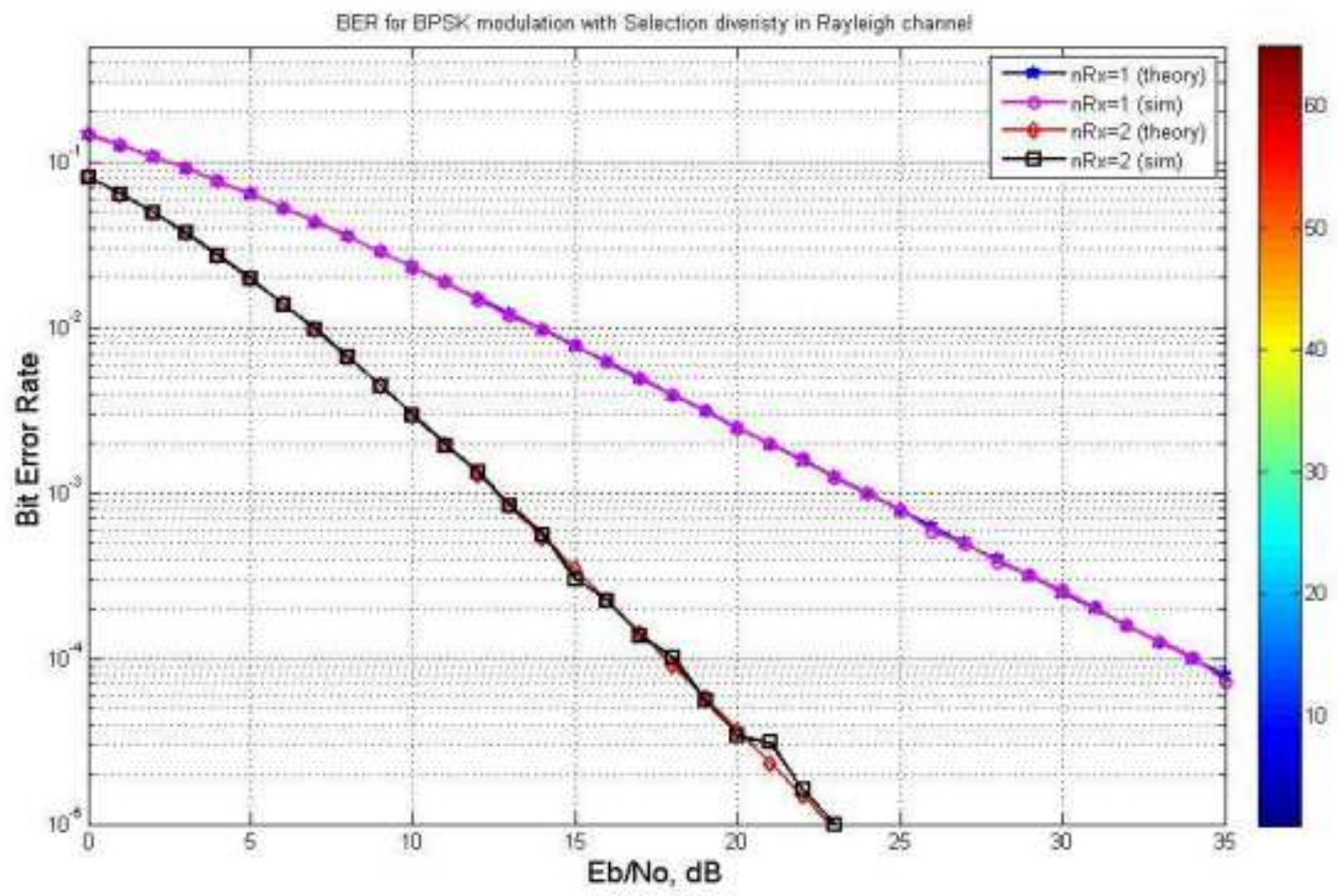

Figure 7

BER for the binary phase-shift keying modulation in a Rayleigh fading channel with selection diversity. 


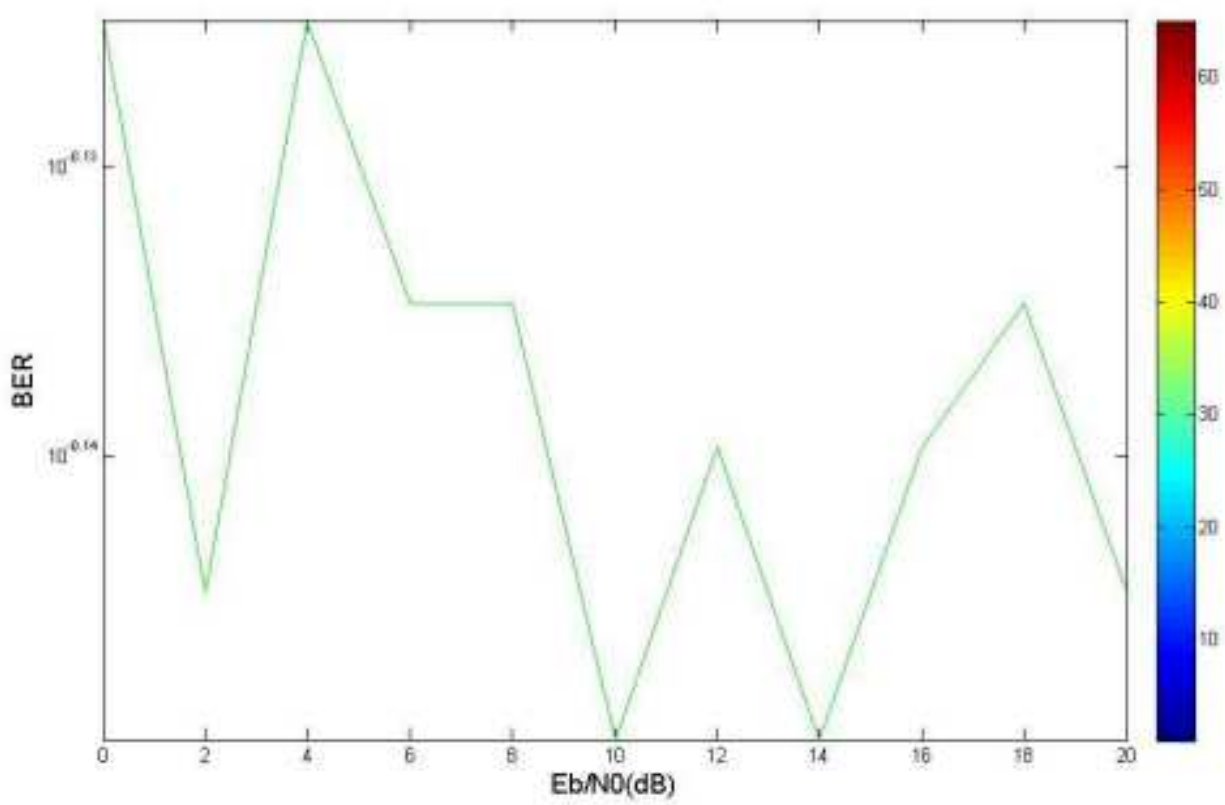

Figure 8

[Please see the manuscript file to view this figure caption.]

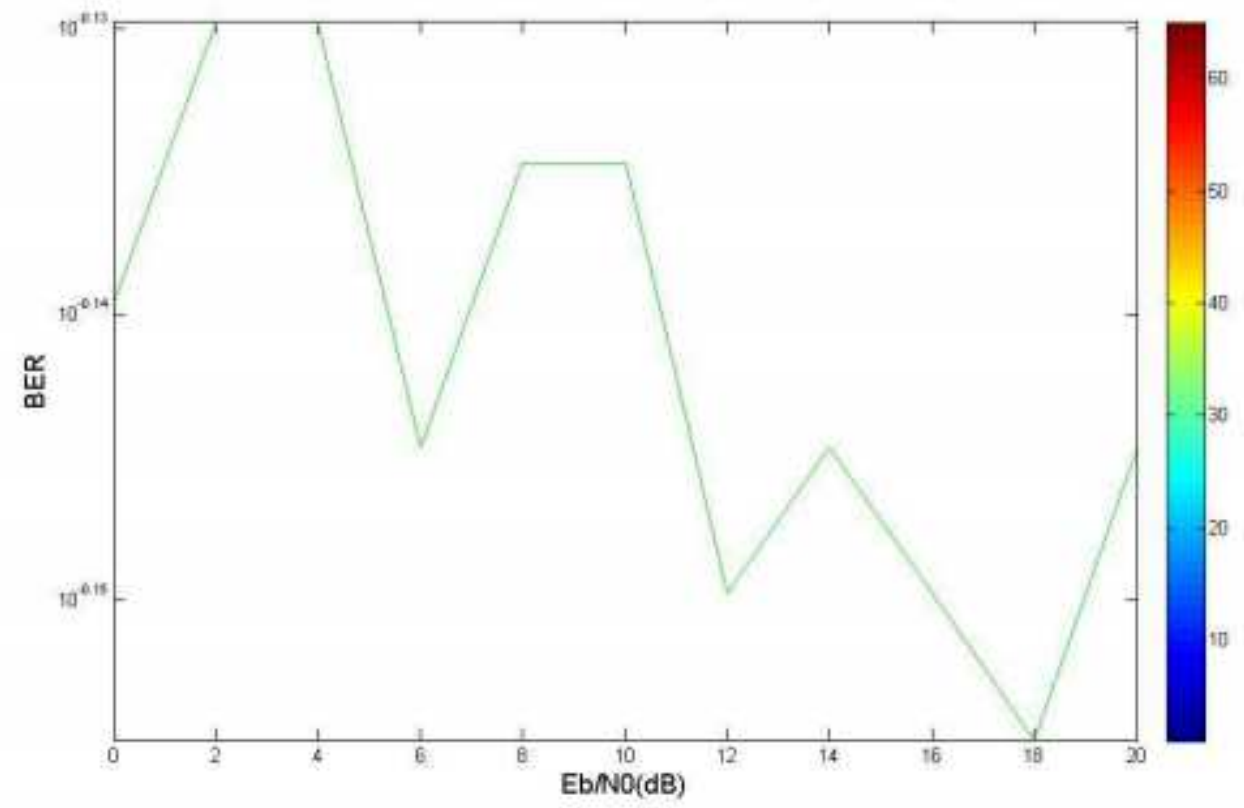

Figure 9

[Please see the manuscript file to view this figure caption.] 


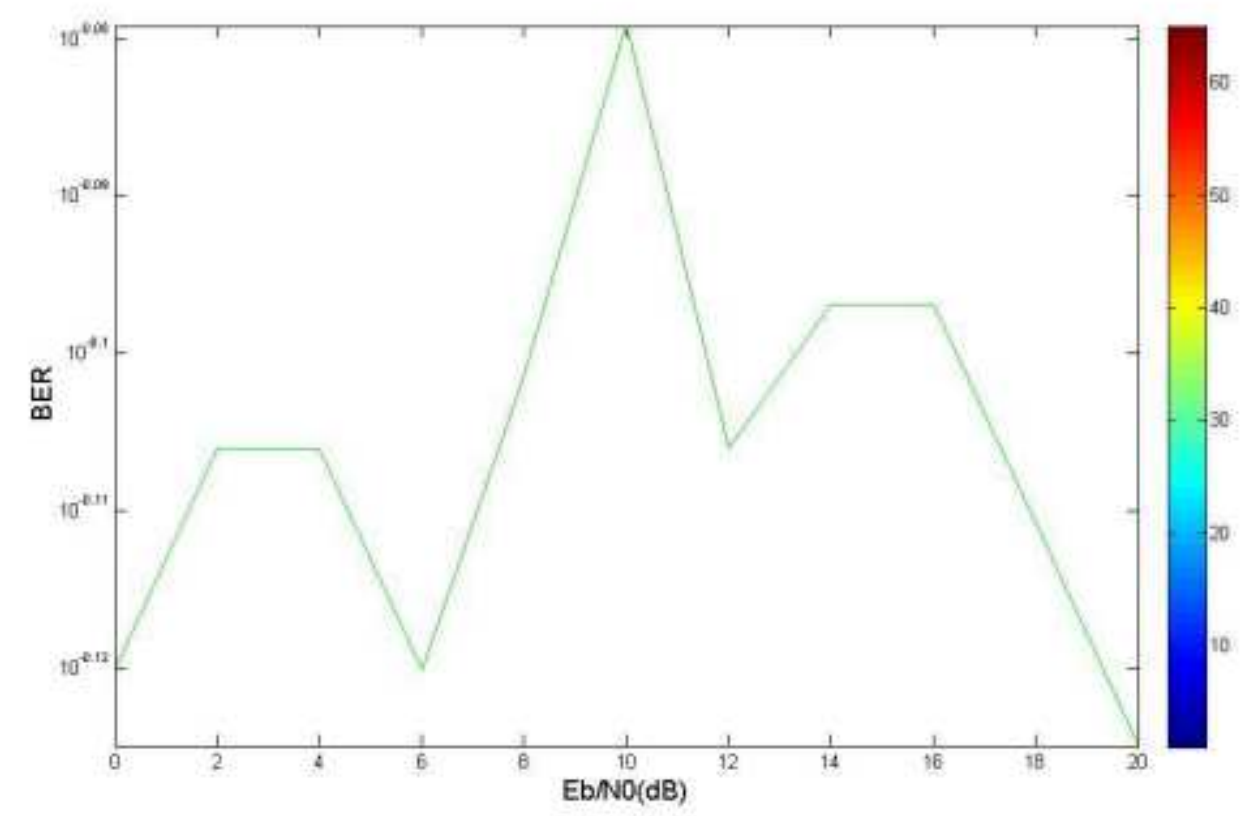

Figure 10

[Please see the manuscript file to view this figure caption.]

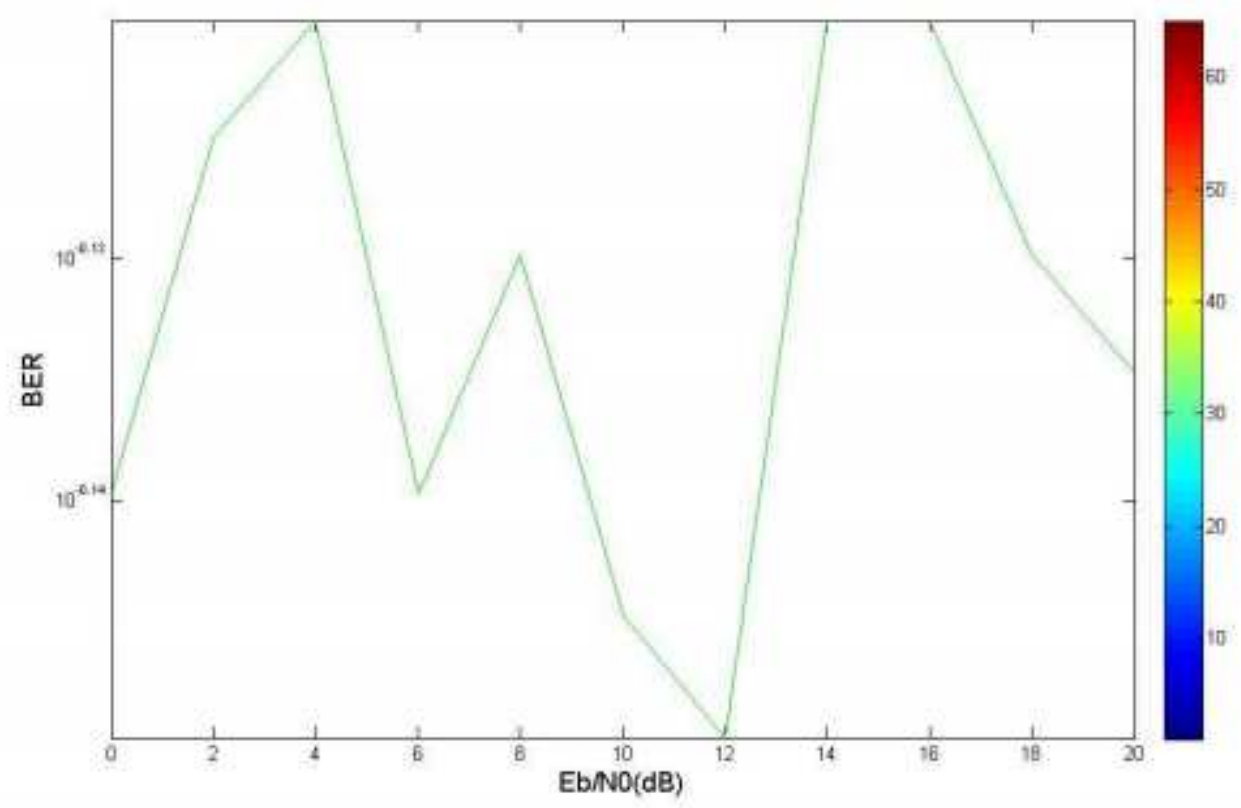

Figure 11

[Please see the manuscript file to view this figure caption.] 